\title{
THE APRIL MEETING IN WASHINGTON
}

The four hundred fifty-eighth meeting of the American Mathematical Society was held at George Washington University, Washington, D.C., on Friday and Saturday, April 28-29, 1950. Registration headquarters were in the Hall of Government, and all sessions were held there. Hotel headquarters were at the Roger Smith Hotel. The attendance was about 200, including the following 168 members of the Society:

J. C. Abbott, R. D. Anderson, Miriam C. Ayer, R. P. Bailey, Joseph Barnett, P. T. Bateman, F. D. Bateman, T. J. Benac, Stefan Bergman, E. E. Betz, R. H. Bing, Garrett Birkhoff, D. H. Blackwell, W. A. Blankinship, J. H. Blau, Joseph Blum, R. P. Boas, T. A. Botts, J. R. Bowman, Jean M. Boyer, N. A. Brigham, F. E. Browder, Charlotte Brudno, Helen J. Buck, R. S. Burington, G. H. Butcher, B. F. Cheydleur, K. L. Chung, Randolph Church, A. H. Clifford, Natalie Coplan, Mary J. Cox, H. S. M. Coxeter, G. F. Cramer, A. R. Craw, J. M. Danskin, W. S. Dawkins, J. B. Diaz, C. H. Dowker, Yael N. Dowker, D. M. Dribin, W. D. Duthie, R. P. Eddy, Samuel Eilenberg, H. E. Ellingson, J. H. Engel, Bernard Epstein, G. C. Evans, William Feller, N. J. Fine, Wilhelm Fischer, E. E. Floyd, Bernard Friedman, H. M. Gehman, B. C. Getchell, Leonard Gillman, H. F. Gingerich, Michael Goldberg, Leon Goldstein, R. A. Good, E. C. Gras, T. N. E. Greville, Emil Grosswald, D. W. Hall, Philip Hartman, G. G. Harvey, R. M. Hayes, E. C. Higgison, H. A. Hill, L. Aileen Hostinsky, W. R. Hydeman, M. A. Hyman, S. B. Jackson, M. P. Jarnagin, Jr., Herbert Jehle, Walter Jennings, R. E. Johnson, F. E. Johnston, M. L. Juncosa, D. E. Kibbey, J. W. Kitchens, V. L. Klee, J. R. Kline, Paul Kopp, W. D. Lambert, R. E. Langer, Richard Latter, W. S. Lawton, R. A. Leibler, W. W. Leutert, J. H. Levin, D. C. Lewis, F. W. Light, Miriam A. Lipschutz, Lee Lorch, E. J. McShane, H. M. MacNeille, C. J. Maloney, Murray Mannos, M. H. Martin, W. T. Martin, Florence M. Mears, A. E. Meder, Jr., T. W. Moore, T. S. Motzkin, S. B. Myers, David Nelson, Paolo Nesbeda, Moris Newman, T. E. Oberbeck, R. E. O'Donnell, M. W. Oliphant, K. L. Palmquist, A. J. Penico, Harry Polachek, R. R. Reynolds, R. P. Rich, G. de B. Robinson, Murray Rosenblatt, J. H. Rosenbloom, P. C. Rosenbloom, H. E. Salzer, S. S. Saslaw, Max Sasuly, G. E. Schweigert, C. W. Seekins, Atle Selberg, Daniel Shanks, George Shapiro, Seymour Sherman, Ruth W. Shnider, D. T. Sigley, R. C. Simpson, A. C. Smith, D. C. Spencer, Vivian E. Spencer, W. J. Strange, G. R. Strohl, E. G. Swafford, Olga Taussky-Todd, William Clare Taylor, J. H. Taylor, Feodor Theilheimer, D. L. Thomsen, John Todd, Miriam M. Torrey, C. A. Truesdell, J. L. Vanderslice, A. H. Van Tuyl, Oswald Veblen, M. C. Waddell, J. L. Walsh, J. V. Wehausen, F. M. Weida, Alexander Weinstein, F. J. Weyl, C. R. White, P. M. Whitman, W. F. Whitmore, G. T. Whyburn, K. G. Wolfson, M. A. Woodbury, Arthur Wouk, J. G. Wozencraft, J. W. Wrench, J. W. Young, P.W. Zettler-Seidel, J. A. Zilber.

The Committee to Select Hour Speakers for Eastern Sectional Meetings invited two speakers. On Friday, April 28, at 4:15 P.M., Dr. R. P. Boas, Executive Editor of Mathematical Reviews, gave an address on Trigonometric extremal problems. Professor G. C. Evans presided. At 2:15 P.M. on Saturday, April 28, Dr. Atle Selberg of the 
Institute for Advanced Study gave an address on The prime number theorem for generalized primes and elementary methods. President J. L. Walsh presided.

Sections for contributed papers were held at 2:00 P.M. and 3:00 P.M. on Friday and at 10:00 A.M. and 11:00 A.M. on Saturday. The presiding officers for these sessions were Professor William Clare Taylor, Professor G. T. Whyburn, Dr. Seymour Sherman, and Dr. J. V. Wehausen.

A resolution of thanks to George Washington University and to the Committee on Arrangements was moved by Professor G. de B. Robinson and adopted by those present at the session held at 2:15 P.M. on Saturday.

The Council met at 8:15 P.M. on April 28.

The Secretary announced the election of the following one hundred and thirty-eight persons to ordinary membership in the Society:

Professor Forrest Edwin Adams, University of Miami;

Mr. Mohammad Wassel Al-Dhahir, Columbia University;

Mr. Samuel I. Altwerger, Newtown High School and New School of Social Research, New York, N.Y.;

Professor Emil Amelotti, Villanova College;

Mr. William Edward Andrus, Jr., International Business Machines Corp., Endicott, N.Y.;

Professor Carolyn Ruth Bahous, Lynchburg College;

Mr. Dean Clifton Benson, Sioux Falls College;

Mr. Morris Carl Bergen, Citrus Junior College, Azusa, Calif.;

Mr. Herbert Ashley Bernhard, Columbia University;

Mr. John Emory Berterman, Cornell University;

Mr. Stanley Francis Block, University of Maryland;

Mr. Russell Newton Bradt, University of Kansas;

Dr. Abraham Brind, New York, N.Y.;

Mr. Walter Clarke Brown, University of Oklahoma;

Mr. Howard Walker Burnette, University of Georgia;

Mr. John Richard Byrne, University of Washington;

Professor Margaret C. Byrne, Saint Joseph's College, Brooklyn, N.Y.;

Professor Harry Nelson Carter, University of Tulsa;

Mr. Ernest Charles Casale, Temple University;

Mr. Harold Hsiao-Li Chang, Princeton, N.J.;

Mr. Robert Francis Clarke, United States Weather Bureau, Miami Springs, Fla.;

Mr. James William Cooley, Woodside, N.Y.;

Mr. Arthur Herbert Copeland, Jr., University of Michigan;

Mr. Hewitt David Crane, International Business Machines Corp., New York, N.Y.;

Professor Mary Honor Cummings, Rhode Island State College;

Mr. Peter M. Curran, Fordham University;

Mr. Max Alexander Dengler, Midwest Research Institute, Kansas City, Mo.;

Brother V. Dominic, Saint Mary's College, Calif.;

Mr. Edwin Richard Dunkle, Jr., Albright College;

Mr. Ralph Edwin Ekstrom, Westminster College, Fulton, Mo.; 
Mr. Irving Jacob Epstein, Evans Signal Laboratory, Belmar, N.J.;

Mr. Walter Feder, City College, New York, N.Y.;

Professor Michael Fekete, Hebrew University, Jerusalem;

Commander Reid Puryear Fiala, United States Navy, Destroyer Division 602;

Professor Emery Louis Gerecz, Vincennes University;

Mr. Myron Leonard Greenberg, University of Michigan;

Mr. Urban S. Greene, Binghamton, N.Y.;

Mr. Joseph Arthur Greenwood, Manhattan Life Insurance Company, New York, N.Y.;

Professor Simon Gruenzweig, Lincoln University, Pa.;

Mr. Jack Cogswell Gysbers, University of Oregon;

Mr. William Bromley Hall, Jr., Long Island City, N.Y.;

Professor Wame Jacob Hallmark, San Antonio College;

Mr. Francis Robert Halpern, Bell Aircraft Company, Buffalo, N.Y.;

Mr. John Charles Harden, Jr., Clemson College;

Mrs. A. Z. Hays, Abilene Christian College;

Mr. Glenn Fairbanks Hewitt, Wright Branch, Chicago City Junior College;

Mr. Theodore Ware Hildebrandt, Massachusetts Institute of Technology;

Professor Philip Murray Iloff, Chico State College, Chico, Calif.;

Sister Mary Amata Kadlack, Saint Joseph's College for Women, Brooklyn, N.Y.;

Mr. Surindra Nath Kalra, University of Illinois;

Dr. Rosella Kanarik, University of Southern California;

Mr. Costas Kassimatis, University of Toronto;

Mr. J. Lawrence Katz, Brooklyn, N.Y.;

Professor Jack Thurston Kent, Agricultural \& Mechanical College, College Station, Tex.;

Miss Muriel Clarissa King, Georgia State College;

Mr. Edward Harvey Kingsley, Roosevelt College, Chicago, Ill.;

Mr. Joseph Klein, Redbank, N.J.;

Mr. Robert J. Koch, New Orleans, La.;

Professor Motokiti Kondô, Kyûsyû University, Japan;

Professor Edward Michael Kovach, Cathedral College, New York, N.Y.;

Mr. Vernon Kuykendall, Clark Junior College, Vancouver, Wash.;

Mr. John Cornelius Laffan, Jr., Seton Hall College, South Orange, N.J.;

Mr. Orlie William Laing, Pasadena City College;

Mr. Seymour Landau, University of Connecticut;

Mr. Serge Lang, Princeton University;

Mr. Richard Kenneth Lashof, New York, N.Y.;

Mr. Richard F. Link, University of Oregon;

Professor Harry Maurice Linnette, Virginia State College;

Mr. Charles K. Loomis, Jr., Detroit Institute of Technology;

Mr. Robert Duncan Luce, Massachusetts Institute of Technology;

Dr. Robert Hastings McCobb, New Jersey State Teachers College, Glassboro, N.J.;

Professor Richard Joseph McCullough, Mount Saint Mary's College, Emmitsburg,

Md.;

Miss Clarice Irene MacDonald, University of Washington;

Professor Duncan MacRae MacEwen, City College, New York, N.Y.;

Dr. Lawrence Earl Malvern, Carnegie Institute of Technology;

Mr. Robert Louis Marceau, University of Kansas;

Mr. Henry George Mazurkiewicz, University of Michigan; 
Mr. José Carlos Mello E. Souza, Rua Redentor 92, Distrito Federal, Brazil; Captain George Brown Miller, Jr., Georgia Military Academy, College Park, Ga.; Professor James L. Miller, Villanova College;

Mr. Robert Elsworth Montgomery, University of Illinois;

Mr. John Coleman Moore, Brown University;

Miss Marian Alease Moore, Purdue University;

Dr. Charles Edward Mullan, Duquesne Light Company, Pittsburgh, Pa.;

Mr. George Max Muller, General Electric Company, Richland, Wash.;

Professor Sigurd Mundhjeld, Concordia College, Moorhead, Minn.;

Mr. James Henry Nash, Fordham University;

Mr. Abas Beaucan Neale, Naval Aircraft Factory, Philadelphia, Pa.;

Miss Elizabeth Jane Neiderberger, Purdue University;

Mr. Ralph Henry Niemann, Purdue University;

Dr. Fritz Oberhettinger, California Institute of Technology;

Mr. Edward Scott O'Keefe, University of Washington;

Miss Katharine Louisa Parks, Morgan Park Junior College, Chicago, Ill.;

Mr. Paul Bryan Patterson, University of Florida;

Mr. Paul Edwin Pfeiffer, Rice Institute;

Professor Harold David Reese, Drexel Institute of Technology;

Mr. Henry Gordon Rice, Syracuse University;

Professor Ralph A. Rodefer, Michigan College of Mining \& Technology;

Mr. Jack Cree Rogers, Cornell University;

Miss Mary Lee Rogers, University of Washington;

Miss Florence Virginia Rohde, University of Kentucky;

Mr. Richard Irwin Rossbacher, New Haven, Conn.;

Mr. George Xavier Saltarelli, Buffalo, N.Y.;

Mr. James Sanders, Syracuse University;

Mr. Frank Salvatore Scalora, University of Illinois;

Professor Olive Crosbie Schooler, University of Tulsa;

Mr. William Howard Sellers, West Virginia University;

Miss Edna Sheinhart, University of Connecticut;

Mr. Alan B. Showalter, University of Kansas;

Professor Marvin Banks Sledd, Emory University;

Professor Richard Herbert Somers, University of New Hampshire;

Mr. William Kebren Spears, Keesler Air Force Base, Biloxi, Miss.;

Mr. Clifford Harvey Springer, Purdue University;

Mr. Jeremiah Milton Stark, Massachusetts Institute of Technology;

Mr. Gordian William Stremlau, St. Bede College, Peru, Ill.;

Professor Mary Virginia Sunseri, Stanford University;

Professor Victor G. Szebehely, Virginia Polytechnic Institute;

Professor Choy-tak Taam, University of Missouri;

Mr. Eugene Titus, Southern University and Agricultural and Mechanical College;

Mr. John William Toole, University of Illinois;

Mr. Grover Jackson Trammell, Jr., Tulane University of Louisiana;

Mr. Charles Hood Tyler, Lewis College, Lockport, Ill.;

Mr. Lewis Edes Ward, Jr., Tulane University of Louisiana;

Mr. Hans Felix Weinberger, Carnegie Institute of Technology;

Professor Annye Elizabeth Welch, Catawba College, Salisbury, N.C.;

Mr. Burton Garland West, Lincoln High School, Port Arthur, Tex.;

Professor John Elliott Westberry, Texas College; 
Mr. Carl Edgar Weyland, College of South Jersey;

Professor Royal Samuel Weymouth, Idaho State College;

Mr. Robert Franklin Wheeling, Brown University;

Professor Clarence Reed White, Hampton Institute;

Mr. James Whittaker, University of California at Los Angeles;

Professor Roy Edwin Wild, University of Idaho;

Professor Ernest Williams, Alabama Polytechnic Institute;

Mr. Wilber Albert Wilson, Clemson College;

Mr. Henry Wolf, Brown University;

Professor Guy Aytch York, Georgia School of Technology;

Mr. Solomon Abraham Zadoff, Passaic, N.J.

It was reported that the following twenty-five persons had been elected to membership on nomination of institutional members as indicated:

Indiana University: Mr. Donald Henry Porter;

Institute for Advanced Study: Dr. Ernest Paul Specker;

University of Minnesota: Messrs. Martin Samuel Friberg and Arnold Jackson Tingley;

Northwestern University: Messrs. Donald Guy Austin, Robert Langley Sternberg, and Fred Marion Wright;

Princeton University: Messrs. Iain Thomas Arthur Carpenter Adamson, Jose Adem Chahin, Richard Charles Blanchfield, Eugenio Calabi, Francis Brock Fuller, John McCarthy, John Willard Milnor, John Forbes Nash, Jr., Dr. Christos Dimitriov Papakyriakopoulos, Messrs. Melvin Philip Peisakoff, Oscar Seymour Rothaus, Joseph Harold Sampson, Jr., Lloyd Stowell Shapley, William Tout Sharp, George Stephenson, John Torrence Tate, Jr., and Guillermo Torres Diaz;

University of Wichita: Professor Cecil Byron Read.

The Secretary announced that the following had been admitted to the Society in accordance with reciprocity agreements with various mathematical organizations: London Mathematical Society: Mr. Cecil John Alvin Evelyn, London; Professor Werner Wolfgang Rogosinski, Durham University and Agricultural and Mechanical College, Stillwater, Okla.; Matematisk Forening, Kфbenhavn: Mr. Bent Fuglede, Stanford University; Polish Mathematical Society: Professor Edward Arnold Otto, Warsaw Politechnike School; Miss Wanda Szmielew, University of California; Société Mathématique de France: Mr. Marcel Honore Bayard, Sous-Marino Telecommunications, Meudon; Dr. Jean Francois Braconnier, University of Lyon; Professor Jovan Karamata, University of Belgrade; Professor Georges de Rham, University of Geneve and Institute for Advanced Study; Mr. Dov Tamari, Boursier de l'A.R.S., Paris; Professor Miodrag Tomic, Belgrade Polytechnic Institute; Swiss Mathematical Society: Professor Hans Georg Haefeli, Boston College; Unione Matematica Italiana: Professor Francesco Elbano Sbrana, University of Genova. 
Texas Christian University (Fort Worth, Texas) was elected to institutional membership in the Society.

The following actions taken by mail vote of the Council were reported: election of Professors Einar Hille and A. W. Tucker as members of the Executive Committee of the Council to serve for a period of two years beginning January 1, 1950; election of Professor W. T. Martin as a representative of the Society on the Policy Committee for Mathematics, to serve for a period of four years beginning January 1,1950; authorization of the President to appoint a committee to study the question of reciprocity agreements with organizations on the American continents; authorization of the President to appoint a committee to cooperate with the American Institute of Graphic Arts in a study of printing problems.

The following appointments of representatives of the Society were reported: Professor R. G. Helsel at centenary celebration of University of Dayton on March 16, 1950; Professor Gillie A. Larew at inauguration of Orville Wentworth Wake as President of Lynchburg College on April 25, 1950; Professor S. P. Shugert at inauguration of H. Sherman Oberly as President of Roanoke College on April 14, 1950; Professor Helen Barton at inauguration of Dale Hartzler Gramley as President of Salem College and Academy on April 22, 1950; Professor V. H. Tingey at 100th anniversary of University of Utah on February 27-28, 1950; Professor Eric Reissner on Program Committee for 1951 London Conference in Heat Transmission (sponsored by American Society of Mechanical Engineers and Institute of Mechanical Engineers, London).

The following additional appointments by the President were reported: Professors T. F. Cope and B. P. Gill as auditors of the Society's accounts for 1950; Professors R. E. Langer (Chairman), J. L. Doob, M. H. Heins, T. H. Hildebrandt, and D. C. Spencer as a Committee to Study Individual Membership Dues; Professor R. L. Wilder as Chairman of the Committee on Places of Meetings for 1950; Professor J. M. Thomas as a member of the Committee on Places of Meetings for 1950-1952; Professors G. T. Whyburn (Chairman), Nelson Dunford, R. L. Jeffery, C. C. MacDuffee, and D. C. Spencer as a Committee to Study the Question of Reciprocity Agreements with Organizations on the American Continents; Professors Saunders MacLane (Chairman), C. C. MacDuffee, G. B. Price, A. C. Schaeffer, and J. M. Thomas as a Committee to Discuss Possible Reductions in Cost of Mathematical Composition; Professors S. S. Cairns (Chairman), E. F. Beckenbach, Einar Hille, Deane Montgomery, and R. L. Wilder as a Committee to Nominate Officers and 
Members of the Council for 1951.

The following resolution on the death of Professor Virgil Snyder was adopted by the Council:

The Council of the American Mathematical Society wishes to record its deep sense of loss in the death of Virgil Snyder, Emeritus Professor at Cornell University since 1938. He had been connected with Cornell University as a member of the faculty since 1895.

Professor Snyder had an active and influential part in the affairs of the Society for fifty years, having served at various times as a member of the Council, vice president, president, as an editor of the Bulletin, and as a delegate to several international congresses.

Professor Snyder's mathematical interest was in the field of algebraic geometry. His graduate study at Göttingen and his later study and association with the Italian geometers laid the foundation for his own outstanding work in this field and for his inspiring guidance of some forty graduate students in the preparation of their doctoral dissertations. His standing in this particular field was recognized when he was selected as chairman of the committee of the National Research Council to prepare a bibliography for algebraic geometry.

During the first half of the twentieth century American interest in mathematics and American contributions to the development of the subject had a phenomenal growth. Now we are called upon once more to note the passing of one of the men who contributed largely to this advance. We do so with sincere appreciation of the man and of his work.

Dr. H. M. MacNeille, Executive Director, reported on the following problems: (1) that of obtaining suitable office space for the Society in New York and the related problem of the use of the Society's library as a bargaining asset in negotiations with Columbia University for office space; (2) that of establishing a new agreement with Johns Hopkins University in connection with the publication of the American Journal of Mathematics. The Council voted to recommend a change in the by-laws to reduce to two the number of representatives of the Society on the Editorial Board of the American Journal; (3) the Executive Director, at the request of the Board of Trustees, had gathered a list of candidates for the position of Executive Editor of Mathematical Reviews, for the consideration of the Editorial Committee.

The Council voted to approve the recommendation of the Committee on Applied Mathematics to hold the 1951 Symposium in Applied Mathematics at the University of Maryland and the United States Naval Ordnance Laboratory.

The Council voted to recommend to the Board of Trustees that Dr. J. V. Wehausen be appointed as Executive Editor of Mathematical Reviews, to succeed Dr. R. P. Boas.

The Policy Committe for Mathematics reported progress in its consideration of the following problems: the establishment of a 
Mathematics Foundation to receive and administer funds for the general support of mathematics; National Science Foundation and the service such a foundation could render to mathematics; separate division for mathematics in the National Research Council; Union Conference to be held at Columbia University on August 27-29, 1950 , for a discussion of a new International Mathematical Union.

The Council adopted a budget for the International Congress as recommended by the Organizing Committee; total estimated expenses for the Congress are $\$ 101,516.00$.

It was reported that the dictionary containing a list of words useful in reading Russian mathematical articles and about thirty pages of grammar would shortly be ready for distribution. The Council voted to recommend to the Trustees that a thousand copies, in addition to the number required by the Navy under the contract, be printed for possible future sales. It was also reported that fifteen translations of Russian mathematical articles had been completed and that the Navy contract for the preparation of these translations had been extended to April 30, 1951.

The Council voted to recommend to the Trustees that for 1951 the list price of the Bulletin be set at $\$ 7.00$ and that of the Proceedings at $\$ 11.00$.

Abstracts of the papers read follow below. Papers whose abstract numbers are followed by the letter " $t$ " were read by title. Paper number 302 was read by Professor Fine, paper number 313 by Professor Epstein, paper number 335 by Dr. Rosenblatt, and paper number 338 by Professor Floyd. Mr. Hurley was introduced by Dr. H. W. E. Schwerdtfeger.

\section{Algebra And Theory of Numbers}

300t. Bailey Brown and N. H. McCoy: Some theorems on groups with applications to ring theory.

Let $G$ be an additively written group, and $F$ a mapping of $G$ into the set of subgroups of $G$. Under certain restrictions on $F$ relative to a set of endomorphisms of $G$, a normal subgroup $N$ is defined, depending on $F$. When $G$ is the additive group of a ring $R, N$ becomes for special choices of $F$, respectively: (1) the greatest regular ideal in $R$ (Bull. Amer. Math. Soc. Abstract 55-3-100), (2) the Jacobson radical of $R$, (3) the $F$-radical of $R,(4)$ the radical of $R$ (Amer. J. Math. vol. 69 (1947) pp. 46-58). Regularity is defined in, and (1) extended to, an arbitrary naring, and a proof is indicated that a full matrix naring over a regular naring is regular. By strengthening the assumptions on $F, N$ becomes for any $G$ the intersection of a certain class of normal subgroups of $G$, and a subdirect decomposition of $G$ is obtained if $N=0$. When $G$ is the additive group of $R$, the corresponding theorems for (2), (3), (4) now become special cases. Here R may be a naring or even a cluster. (Received March 27, 1950.) 


\section{A. H. Clifford: A noncommutative simple linearly ordered group.}

In answer to Garrett Birkhoff's Problem 5 (Ann. of Math. vol. 43 (1942) p. 329) an example is given of a noncommutative linearly ordered group having no proper $l$-ideals. (Received March 14, 1950.)

302. N. J. Fine and G. E. Schweigert: Concerning the group of homeomorphisms of a simple arc.

Let $G$ denote the group of all homeomorphisms of a simple arc onto itself. It is shown that every element of $G$ is a product of at most four involutions (elements of order two). Certain subgroups of $G$ are characterized, and $G$ itself is determined among them by a maximality condition. A partial identification of the normal subgroups is made. An interesting class of commutative subgroups is studied in some detail, with special reference to their intersection properties. Problems similar to those above are considered for other (possibly nonseparable) spaces with order topologies. The results on $G$ can be used to show that every element in the homeomorphism group of a simple closed curve is representable as a product of at most five involutions. (Received March 9, 1950.)

\section{L. Aileen Hostinsky: Direct decompositions in lattices.}

The refinement theorem proved by R. Baer for direct decompositions of operator loops (Direct decompositions, Trans. Amer. Math. Soc. vol. 62 (1947) pp. 62-98) is generalized by using results in the author's Bull. Amer. Math. Soc. Abstract 55-11-474 to transla te into lattice theory Fitting's method of investigating refinement theorems. Relationships between this result and theorems of O. Ore, A. Kurosch, and M. Grayev are shown. (Received February 14, 1950.)

\section{4t. A. C. Hurley: The irreducible crystal classes in four dimensions.}

The problem of finding the crystal classes is tackled using the theory of group characters. Firstly all the types of elements which may occur are determined using the fact that the characteristic equation of the matrix must have integral coefficients (the invariants of the matrix). The second invariants of the matrices are shown themselves to constitute the character of a representation of the group: thus in using the result of character theory, that all power sums of the characters give totals which are multiples of the order of the group, the second invariants may be used in forming the power sums as well as the characters. Using this method the possible values for the order of a four-dimensional crystal group are found, as are also the number of elements in each group having a given value of the trace, second invariant, and determinant. Of these solutions of the character relations, some (38) are shown to represent irreducible crystal groups (derived from the regular four-dimensional polytopes and other methods), some are shown to be extraneous, whilst some (33) remain unaccounted for. (Received April 11, 1950.)

\section{5t. A. C. Hurley: The crystal classes in four dimensions.}

The investigation is based on a paper by Goursat (Ann. l'Êcole Norm. vol. 16 (1889)). In this paper Goursat determines all finite four-dimensional rotation groups which contain $-I$, where $I$ is the unit element. The trace and second invariant of all elements of Goursat's groups are determined, those groups with non-integral invariants being discarded. Of the remaining groups most are shown to be crystal 
groups. Some however are not; these may be eliminated using the results of a paper by Robinson (Proc. Cambridge Philos. Soc. (2) vol. 32 (1930) p. 12). The crystal groups not containing $-I$ are also found. The results of the character theory investigation given in the preceding abstract provide a useful check on the final list of groups, and in fact lead to the detection of an error of omission in the above-mentioned paper by Goursat. Using these methods it is shown that there are just 218 four-dimensional crystal groups, of which 45 are irreducible. (Received April 11, 1950.)

\section{6t. D. J. Newman: An evaluation of the constant in the asymptotic expression for $p(n)$.}

Hardy and Ramanujan proved in 1918 the asymptotic formula $p(n)$ $\sim\left(1 / 4\left(3^{1 / 2}\right)\left(e^{\pi(2 n / 3)^{1 / 2}} / n\right)\right.$, where $p(n)$ represents the number of partitions of $n$ (Asymptotic formulae in combinatory analysis, Proc. London Math. Soc. (1918) pp. 75-115). Erdös in 1942 proved, by elementary methods, that $p(n) \sim a e^{\pi(2 n / 3)^{1 / 2}} / n$ for some $a>0$, but did not prove that $a=1 / 4\left(3^{1 / 2}\right)$ (On an elementary proof of some asymptotic formulas in the theory of partitions, Ann. of Math. (1942) pp. 417-430). In this paper the author proves that $a=1 / 4\left(3^{1 / 2}\right)$ by elementary methods. The method used is to estimate the two series $\sum p(n) x^{n} \sim\left(e^{-\pi^{2} / 12} /(2 \pi)^{1 / 2}\right)(1-x)^{1 / 2} e^{\pi^{2} / 6(1-x)}$ and $\sum\left(e^{\pi(2 n / 3)^{1 / 2}} / n\right) x^{n} \sim 2 e^{-\pi^{2} / 12}(6 / \pi)^{1 / 2}(1-x)^{1 / 2} e^{\pi^{2} / 6(1+x)}$ from which an immediate application of the theorem $A_{n} \sim a B_{n} \rightarrow \sum A_{n} x^{n} \sim a \sum B_{n} x^{n}$ gives $\left(e^{-\pi^{2} / 12} /(2 \pi)^{1 / 2}(1-x)^{1 / 2}\right.$ $\cdot e^{\pi^{2} / 6(1-x)} \sim a 2 e^{-\pi^{2} / 12}(6 / \pi)^{1 / 2} e^{\pi^{2} / 6(1-x)}$ and this proves $a=1 / 4\left(3^{1 / 2}\right)$. (Received March 6, 1950.)

\section{R. P. Rich: On partially ordered groups.}

A compositive convex normal subgroup of a partially ordered group $G$ is called an $l$-ideal; this reduces to Birkhoff's definition (Ann. of Math. vol. 43 (1942) p. 310) if $G$ is lattice ordered. The set $\&$ of $l$-ideals of $G$ is a complete lattice under inclusion. If $G$ has the Riesz interpolation property (Birkhoff, loc. cit. p. 328), then $\&$ is a distributive sublattice of the lattice $\mathfrak{R}$ of normal subgroups of $G$, and the complemented $l$-ideals in $\&$ form a Boolean algebra. Answering Birkhoff's Problem 5 (loc. cit. p. 329): there exists a non-archimedean lattice ordered group $H$ which is simple, that is, without proper $l$-ideals; $H$ is a subgroup of the group of homeomorphisms of an interval considered by Everett and Ulam (Trans. Amer. Math. Soc. vol. 57 (1945) p. 212). If $C$ is a linearly ordered group with an $l$-ideal $A$ such that $A$ and $C / A$ are both archimedean, then (i) if $A$ is in the center of $C$, the factor set of $C / A$ in $A$ is arbitrary; (ii) if $A$ is not in the center of $C$, then the factor set is associate to the identity. (Received March 14, 1950.)

\section{8t. C. E. Rickart: Isomorphic groups of linear transformations: the unitary and simpletic cases.}

In another paper (to appear in Amer. J. Math., cf. also Bull. Amer. Math. Soc. abstracts 54-11-440, 55-11-487) the structure of isomorphisms of certain groups of linear transformations which reduce in the finite-dimensional case to full linear groups was discussed. In the present paper, a similar investigation is carried out for groups of linear transformations which reduce in the finite-dimensional case to unitary (or orthogonal) groups on the one hand and to simplectic groups on the other. The main result is that the group isomorphisms are essentially generated by isomorphisms of the underlying vector spaces on which the transformations act. The result in the finite-dimensional simplectic case reduces essentially to a result an- 
nounced by Dieudonné (C. R. Acad. Sci. Paris vol. 225 (1947) pp. 914-915). (Received March 20, 1950.)

\section{G. de B. Robinson: Induced representations and invariants.}

Consider the subroup $H=S_{m} \times S_{m} \times \cdots \times S_{m}$ ( $n$ factors) and its normalizer $\mathfrak{R}(H)$ in the symmetric group $S_{m n}$. If $[\alpha]$ is an irreducible representation of $S_{m}$, then the representation $[\alpha]^{n}$ of $H$ can be extended by means of a representation $[\beta]$ of $S_{n}$ to yield an irreducible representation $[\alpha ; \beta]$ of $\Re(H)$, and this representation $[\alpha ; \beta]$ induces a representation $[\alpha] \odot[\beta]$ of $S_{m n}$. In a previous paper the author gave an explicit formula for the reduction of $[\alpha] \odot[\beta]$ in terms of group characters. This formula has since been obtained by J. A. Todd (to appear shortly in the Canadian Journal of Mathematics) via the corresponding theory of the full linear group. However, so far as the applications to invariants are concerned, it would be desirable to avoid the complications arising from such a dependence upon character tables. The present method accomplishes this end through a new "integration" process which makes it possible to pass from $n$ to $n+1$ for a fixed $[\alpha]$. It is necessary to add a "boundary condition" which depends on the $\overline{n+1}$-hook structure of the Young diagrams so obtained. (Received March 20, 1950.)

\section{Olga Taussky: Symmetrization of systems of linear equations.}

Let $A=\left(a_{i k}\right)$ be a real nonsingular $n \times n$ matrix, $\lambda_{\max }, \lambda_{\min }$ its characteristic roots of largest, resp., smallest modulus, $A^{\prime}$ the transpose of $A$ and $\left(b_{i k}\right)$ its inverse. The following two "condition numbers" of $A$ have been introduced recently: (i) $\left|\lambda_{\max }\right| /\left|\lambda_{\min }\right|$; (ii) $(1 / n)\left(\sum a_{i k}^{2}\right)^{1 / 2} /\left(\sum b_{i k}^{2}\right)^{1 / 2}$ (see J. von Neumann and H. H. Goldstine, Bull. Amer. Math. Soc. vol. 53 (1947) pp. 1021-1099, and A. M. Turing, Quarterly Journal of Mechanics and Applied Mathematics vol. 1 (1948) pp. 287-308). It is shown that both numbers increase if $A$ is replaced by $A A^{\prime}$. Thus symmetrization of a system of linear equations, in general, leads to a worsening of the condition. Further, the class of the matrices $A A^{\prime}$ is studied for which (ii) is constant. (Received March 14,1950.)

\section{M. C. Waddell: On properties of regular rings.}

A ring $R$ is regular if, for each $a \in R$, there exists $x \in R$ with $a x a=a$. A regular ring is called $t$-regular if every principal ideal has a unit element, and is called $s$-regular if it contains no nonzero nilpotent element. Several properties, analogous to those of classical semi-simple ring theory, are obtained, including the following. In a regular ring every ideal is the meet of all generalized prime ideals (in the sense of McCoy, Amer. J. Math. vol. 71 (1949) p. 823) containing it, and the ideals form a distributive lattice. In a $t$-regular ring every ideal is the meet of all maximal ideals containing it, and if every maximal ideal has nonzero annihilator, then every ideal contains a minimal ideal, the ring is isomorphic to a subdirect sum of its minimal ideals, and the ideals form a Boolean algebra. Nine necessary and sufficient conditions for a regular ring to be $s$-regular are given, including (i) every idempotent element is in the center of the ring, (ii) only two-sided ideals exist, (iii) every generalized prime ideal is prime, (iv) every ideal is the meet of all prime ideals containing it, (v) every ideal is a radical ideal (that is, $a^{n} \in A$ implies $a \in A$ ). Assuming $s$-regularity, the sets of prime, maximal, and indecomposable ideals coincide. (Received March 14, 1950.)

\section{ANALysis}

312t. R. P. Boas: Differential equations of infinite order. 
The equations are of the form $A(D) f(z)=g(z)$, where $D=d / d z, A(t)=\sum_{0}^{\infty} a_{n} t^{n}$ is an entire function, $g(z)$ is regular in a (sufficiently large) neighborhood of 0 , and the solution $f(z)$ is to be regular in some neighborhood of 0 . By means of exponential transforms [Boas, Proc. Nat. Acad. Sci. U.S.A. vol. 34 (1948) pp. 481-483] some results of Sheffer (Duke Math. J. vol. 3 (1937) pp. 593-609) and Muggli (Comment. Math. Helv. vol. 11 (1938) pp. 151-179) are obtained when $A(t)$ is of exponential type. In addition, it is shown that there is always a solution when $A(t)$ belongs to a wider class of entire functions, whose essential property is that they increase at their maximum rate in at least three directions which are the normals to the sides of a closed convex polygon; $A(t)=\exp \left(t^{3}\right)$ is a typical example. (Received March 15, 1950.)

\section{Joseph Lehner and Bernard Epstein: Behavior of modular functions and forms near the boundary.}

The authors prove the following results, $x$ representing a real number: (1) If $J(\tau)$ is the absolute invariant of the full modular group, and if $x$ is irrational, then along no curve terminating at $x$ does $J(\tau)$ approach a limit, finite or infinite, while it is known that $J(\tau) \rightarrow \infty$ as $\tau$ approaches any rational $x$ in any Stolz angle. (2) If $f(\tau)$ is a modular form belonging to the full group, then $f(\tau)$ possesses no limit at irrational $x$ except on a certain set $E$ where $f(\tau) \rightarrow 0$ or $\infty$ in any Stolz angle according as $f(r)$ is of positive or negative dimension. The set $E$ consists of those irrationals whose expansions in regular continued fractions have bounded partial quotients. (3) These results generalize to certain subgroups of the full group. (4) There exists an angle $\alpha$ independent of $x$ such that, with the exception of a countable set of $x$, the image in the $w$-plane under the mapping $w=\lambda(\tau)$ of the Stolz angle of aperture $\alpha$ with vertex at $x$ covers infinitely of ten a certain domain of the w-plane. (Received March 27, 1950.)

\section{4t. Dorothy Maharam: Decompositions of measure of algebras} and spaces.

If $A$ is a given $\sigma$-subalgebra of a $\sigma$-finite measure algebra $(E, \mu)$, it is shown that $(E, \mu)$ is isometric to a subalgebra of a principal ideal in the direct product of two $\sigma$-finite measure algebras, one of which is algebraically isomorphic to $A$. (Cf. Gleason, Bull. Amer. Math. Soc. Abstract 55-3-144; Nikodým, C. R. Acad. Sci. Paris vol. 228 (1949), for related results when $\mu$ is finite.) This is proved by introducing a suitable abstract-valued measure in $E$ and applying the author's representation theorem (Trans. Amer. Math. Soc. vol. 65 (1949)). Applications are made to normal measure spaces $(\Omega, \mu)$; for example, if $(\Omega, \mu)$ is acted on by a measure-preserving transformation, the direct sum decomposition of $\Omega$ into ergodic parts can be imbedded, in a simple way, in a direct product decomposition. Other results due to von Neumann, Halmos, and Dieudonné are extended similarly. (Received March 10,1950.)

315t. E. F. Moore: Density ratios and $(\phi, 1)$ rectifiability in $n$-space.

If $\phi$ is a linear measure over Euclidean $n$-space $E_{n}$, a set $A \subseteq E_{n}$ is said to be $(\phi, 1)$ rectifiable if and only if for every $\epsilon>0$ there is a rectifiable set $B$ such that $\phi(A-B)<\epsilon$. If $\phi(A)<\infty$, the $(\phi, 1)$ rectifiability of $A$ is shown to be equivalent to inequalities holding for $\phi$ almost all $x$ in $A$ between constant multiples of certain upper and lower density functions defined locally in terms of $\phi$ and $A$. This extends (with the same constants used in the inequalities) results proved in the case $n=2$ by Morse and 
Randolph (Trans. Amer. Math. Soc. vol. 55 (1944) pp. 236-305). (Received April 17, 1950.)

\section{Ruth E. O'Donnell: $k$-polar polynomials.}

The purpose of this paper is to prove a generalization of the theorem of Grace on apolar polynomials. The polynomials $f(z)=\sum_{j=0}^{n} C_{n, j} a_{j} z^{j}$ and $g(z)=\sum_{j=0}^{n} C_{n, j} b_{j} z^{i}$ are $k$-polar if their coefficients satisfy the $k^{2}$ conditions $\sum_{j=0}^{n-k+1}(-1)^{i} C_{n-k+1, j} a_{s-j} b_{j+h}$ $(h=0, \cdots, k-1 ; s=n, \cdots, n-k+1)$. It is proved that the $k$-polarity of two polynomials is sufficient to insure that every circular domain containing all the zeros of one of the polynomials shall contain at least $k$ zeros of the other. (Received March $16,1950$.

\section{7t. J. E. L. Peck: An ergodic theorem for a certain non-commuta-} tive semigroup of linear operators.

If $\mathcal{L}$ is a semigroup of bounded linear operators on a Banach space, which is totally bounded in the strong topology of operators, and which satisfies the pseudo-commutative restrictions (1) given $S_{1}, S_{2}$ in $\mathcal{L}$ there exists $S_{3}, S_{4}$ in $\mathcal{L}$ with $S_{1} S_{3}=S_{2} S_{4}$ and (2) given $S_{5}, S_{6}$ in $\mathcal{L}$ there exist $S_{7}, S_{8}$ in $\mathcal{L}$ with $S_{5} S_{6}=S_{7} S_{5}=S_{6} S_{8}$; then $\mathcal{L}$ is an ergodic semigroup in the sense of Eberlein (Trans. Amer. Math. Soc. vol. 67 (1949) pp. 217-240) and every element of the Banach space is ergodic in the same sense. This theorem is proved by making use of a fixed point theorem of Kakutani (Proc. Imp. Acad. Tokyo vol. 14 (1938) pp. 242-245). (Received April 17, 1950.)

\section{P. C. Rosenbloom: A problem in Hilbert space.}

Let $A$ be a non-negative bounded Hermitian operator, $x_{0}$ a given element in Hilbert space, and $t$ a given positive constant. We seek the maximum of $\left|\left(x, x_{0}\right)\right|$ under the conditions $\|x\|=1$ and $(A x, x)=t$. This maximum and the extremal element $x$ can be determined in terms of the function $F(\lambda)=\left((\lambda I-A)^{-1} x_{0}, x_{0}\right)$, which is a Stieltjes transform. The behavior of the maximum as $t \rightarrow 0$ depends on that of $F(\lambda)$ as $\lambda \rightarrow 0-$. We obtain a known inequality of Carlson as a special case, and also the best possible inequalities for the maximum modulus of an analytic function on a given circle when the mean square on two given circles has prescribed values. Other applications to analytic and harmonic functions are obtained. (Received March 23, 1950.)

319. George Shapiro: On the Dirichlet series associated with Ramanujan's function $\tau(n)$. Preliminary report.

Let $F(s)=\sum_{n=1}^{\infty} \tau(n) n^{-s-11 / 2}$ for $\sigma>2 / 5$. This series, as well as the infinite product representation $F(s)=\prod_{k=1}^{\infty}\left(1-\tau\left(p_{k}\right) p_{k}^{-s-11 / 2}+p_{k}^{-28}\right)^{-1}$, is known to be absolutely convergent when $\sigma>1$; hence, $F(s) \neq 0$ there. Let $G$ be the (simply-connected) region obtained from the half plane $\sigma>1 / 2$ by deleting all horizontal segments $\sigma+i t_{0}$, $1 / 2<\sigma \leqq \sigma_{0}$, where $\sigma_{0}+i t_{0}$ is a zero of $F(s)$ in $\sigma>1 / 2$ (if such exists). By $\log F(s)$ in $\sigma>1 / 2$ is meant the analytic continuation into $G$ of $\log F(s)$ in $\sigma>1$. Let $F_{n}(s)$ $=\prod_{k=1}^{n}\left(1-\tau\left(p_{k}\right) p_{k}^{-s-13 / 2}+p_{k}^{-2 s}\right)^{-1}$. By iteration of the alternate use of a result of Carlson (Arkiv för Matematik, Astronomi och Fysik vol. 19A (1926) no. 25) and the functional equation of $F(s)$, it is shown that $|F(\sigma+i t)|^{2}$ has a finite mean value for every fixed $\sigma>13 / 20$. From this it follows, by a method similar to that of Bohr (Acta Math. vol. 40 (1916) p. 67), that for every fixed $\sigma>13 / 20$, the sequence $\left\{\log F_{n}(s)\right\}$ 
converges in relative measure to $\log F(s)$. On the basis of Ramanujan's conjecture that $\tau(n)=O\left(n^{11 / 2+\epsilon}\right)$, the same results are obtained in $\sigma>1 / 2$. (Received March 14, 1950.)

320. K. G. Wolfson: On the spectrum of a boundary value problem with two singular end points.

Let $p=p(t)>0$ and $q=q(t)$ be continuous functions for $-\infty<t<+\infty$ such that ( $^{*}\left(p x^{\prime}\right)^{\prime}+(q+\lambda) x=0$ is of "Grenzpunkt" type at $t=-\infty$ and $t=+\infty$. Let the spectrum of the boundary value problem thus determined be denoted by $S$, and the set of its cluster points by $S^{\prime}$. For $\epsilon>0$ and $a<b$, let $N(a, b, \lambda, \epsilon)$ be the number of eigenvalues in the interval $[\lambda-\epsilon, \lambda+\epsilon]$ belonging to the Sturm-Liouville problem determined by $\left({ }^{*}\right)$ and the boundary conditions $x(a)=x(b)=0$. Put $n(\lambda, \epsilon)$ $=\lim \sup N(a, b, \lambda, \epsilon)$, as $a \rightarrow-\infty$ and $b \rightarrow+\infty$. Finally, let $\delta\left(\lambda_{0}\right)$ denote the number $(0,1$, or 2$)$ of the half-lines $(-\infty, 0],[0, \infty)$ on which $\left(^{*}\right)$ is oscillatory for $\lambda$ near, but exceeding, $\lambda_{0}$. Then $\lambda_{0}$ is in $S$ if and only if $n\left(\lambda_{0}, \epsilon\right) \geqq \delta\left(\lambda_{0}\right)+1$ for all $\epsilon>0$; while $\lambda_{0}$ is in $S^{\prime}$ if and only if $n\left(\lambda_{0}, \epsilon\right)=\infty$ for all $\epsilon>0$. (Received March 14, 1950.)

321. Arthur Wouk: Difference equations and J-matrices. I. Preliminary report.

Let $J=\left(j_{i k}\right)$ be a real symmetric infinite matrix which is of the Jacobi type, that is, $j_{i k}=0$ if $|i-k|>1$ and $j_{i, i+1}>0$. A connection between $J$ and a Sturm difference equation $\Delta\left(p_{n} \Delta x_{n}\right)+q_{n} x_{n+1}=0$, where $p_{n}=j_{n, n+1}, q_{n}=j_{n n}+j_{n-1, n}+j_{n, n+1}$, is implicit in Hellinger's paper on Continued fraction theory, Math. Ann. vol. 86 (1922) pp. 18-29. Methods common in the theory of the Sturm differential equation are applied to the difference equation to obtain criteria for $J$ to be self-adjoint and to obtain a characterization of the spectrum of $J$. (Received March 14, 1950.)

322t. D. M. Young: The rate of convergence of an improved iterative method for solving the finite difference analogue of the Dirichlet problem.

Using overrelaxation combined with the Liebmann method, the number of iterations required to solve the finite difference analogue of the Dirichlet Problem for an $n$-dimensional network with mesh size $h$ is asymptotically proportional to $h^{-1}$ if the overrelaxation factor $\omega_{1}$ is suitably chosen. This compares with $h^{-2}$ for the Liebmann method. If $\mu_{1}$ is the largest eigenvalue of the Richardson method (Philos. Trans. Roy. Soc. London Ser. A vol. $210(1910)$ pp. 307-357) $\mu_{1}^{2} \omega_{1}^{2}=4\left(\omega_{1}-1\right)$. For a rectangular network with sides $I_{i} h, \mu_{1}=(1 / n) \sum_{i=1}^{n} \cos \left(\pi / I_{i}\right)$. For other regions $\left(1-\mu_{1}\right)$ can be estimated by comparison theorems. If $\left(1-\mu_{1}\right)$ is replaced by $\theta\left(1-\mu_{1}\right)(0<\theta<1)$, the relative increase in the required number of iterations is about $\left(1 / \theta^{1 / 2}-1\right)$. The eigenvalues for the method defined by any overrelaxation factor $\omega \geqq \omega_{1}$ each have absolute value $(\omega-1)$, and the normal form of the matrix is diagonal except if $\omega=\omega_{1}$. If $\omega=\omega_{1}$, the normal form has precisely one non-diagonal element. The formula for $\omega_{1}$ is valid for any self-adjoint second order partial difference equation in two variables. The above statements about the normal matrix form are also valid. (Received March $6,1950$.

\section{Applied Mathematics}

323. J. B. Diaz: Concerning scalar products and certain minimum and maximum principles of mathematical physics. 
Let $A$ and $B$ be points of three-dimensional Euclidean space; $P$ the plane through $B$ perpendicular to the straight line joining $A$ and $B$; and $S$ the sphere through $A$ and tangent to $P$ at $B$. Then (1) (non-negative) minimum principle: for a point $C$ of $P, d(B, A) \leqq d(C, A)$ ( $d$ denotes distance); and (2) (non-negative) maximum principle: for a point $C$ of $S, d(C, A) \leqq d(B, A)$. Simply derived analogues of (1) and (2) for a linear vector space with a positive semi-definite scalar product $(,$,$) yield-$ for various choices of the scalar product-many minimum and maximum principles (some hitherto unknown) in hydrodynamics, elasticity, electromagnetic theory, and so on. All of these are thus seen to have a common origin. As an example, suppose $A$ is the solution of a boundary value problem consisting of a single linear differential equation plus some linear boundary conditions. Four distinct principles for $A$ are obtained. Two of them, one a minimum and one a maximum principle, characterize $A$ among functions satisfying the boundary conditions. The other two, one a minimum and one a maximum principle, characterize $A$ from among the solutions of the differential equation. (Received March 14, 1950.)

\section{M. A. Hyman: Solution of boundary value problems as initial} value problems.

A technique is presented for obtaining numerical solutions of boundary value problems over elementary regions, such as rectangles or rings. The method is applicable whenever a formula $(\mathrm{F})$ giving an exact solution is available. If the differential (or difference) equation is linear and the region $R$ elementary, one can usually obtain (F) by introducing normal or uncoupled coordinates. One evaluates the derivatives (or differences) of $(\mathrm{F})$ on a part $P$ of the complete boundary, then seeks the solution of the differential (or difference) equation in $R$ with initial values given at $P$. This initial-value problem can be solved step-wise with simple operations at each step. Several "initial lines" may be used, on the boundary and inside the region (one motive being to control error growth). Using this method, Laplace's equation (in difference form) has been solved over a rectangle. A solution over a general region may be effected by transforming to an elementary region, provided the corresponding transform of the differential (or difference) equation is tractable. Since this technique, when applied to a linear difference equation, in effect solved a system of linear algebraic equations, it constitutes a new approach to high-order matrix inversion. (Received March 16, 1950.)

325. Herbert Jehle: Complex and real representations of the Lorentz group without reflections.

The relationship between Dirac's and Majorana's wave equations as well as that between the wave equations of Majorana and of the present author are investigated. (Cf. Serpe, Physical Review (1949), Jehle, Bull. Amer. Math. Soc. vol. 56 (1950)). (Received March 15, 1950.)

\section{6t. C. N. Mooers: Generation of unique ciphers for a finite net- work.}

It is desired to assign a unique cipher of letters and numerals to each chemical compound of finite size representable by a network. Such assignment is shown to be possible with a fixed number of rules making a closed set: Cuts are made at arbitrary positions in rings and at branching points to give linear segments. Segments are 
placed end to end in arbitrary order, and are translated into letters and signs by use of conventional chemical abbreviations, hyphens for bonds, and semicolons to indicate breaks. Each character and mark is given a number, starting with one at the left. Connection into molecule of the end of each segment is shown by the corresponding number, zero for a terminal, and these numbers are inserted into expression at ends of each segment. The result is a non-unique cipher. Process is repeated for all possible cuts and orderings, giving set of all possible ciphers for molecule. By an alphabetization of characters, the set of ciphers is ordered, and the first cipher is designated as unique. The large but finite number of steps required is a consequence of the requirement for a fixed number of rules. (Received March 15, 1950.)

\section{7t. Seymour Sherman: Games and sub-games.}

Suppose a two-person zero-sum game matrix $B$ is decomposed into $M N$ submatrices $\left\{B_{j}^{i} \mid 1 \leqq i \leqq M, 1 \leqq j \leqq N\right\}$, where the rows of $B$ involved in the sub-matrix $B_{j}^{i}$ are independent of $j$ and the columns independent of $i$, and that the value of the game associated with $B_{j}^{i}$ is $v_{j}^{i}$. If, for each $i$, there is a common optimal strategy for the first player in the games, $\left\{B_{j}^{i} \mid 1 \leqq j \leqq N\right\}$, then $v$, the value of game associated with $B$, is greater than or equal to $\bar{v}$, the value of game with matrix, $\bar{B}=\left\{v_{j}^{i}\right\}$. Under analogous conditions "down columns" an upper bound for the value of the game and a strategy for the second player preventing the payoff from exceeding the upper bound are given. In the event that both conditions, that is, "across rows and down columns," are satisfied, the value of the game and a set of optimal strategies are determined from the values and optimal strategies of the games, $B_{j}^{i}$, and the value and optimal strategies of the game, $\bar{B}$. (Received February 29,1950 .)

\section{C. A. Truesdell: On Poincarê's analogy between vorticity and mass-density.}

Let $r$ be the radius vector, let its polyadic $n$th power be defined by $r^{(0)} \equiv 1, r^{(n)}$ $\equiv r^{(n-1)} r$, let $w$ be the vorticity vector of a continuous motion, let $\left\{r^{(n)} w\right\}$ stand for $r^{(n)} w+r^{(n-1)} w r+\cdots+w r^{(n)}$. Then $W_{n} \equiv \int\left\{r^{(n)} w\right\} d V$ taken over a finite volume is expressible as an integral over the boundary surface. Hence the vector moments $W_{n}$ are all zero in a motion bounded by finite walls, to which the material adheres without slipping, or in a motion vanishing at $\infty$ to a sufficiently high order; under somewhat weaker conditions, they are constant. These purely kinematical results, generalizing some vortex theorems of Poincaré, exhibit a strong regularity in the average distribution of vorticity in many common types of three-dimensional motions. The conditions for their validity are never satisfied by a plane motion. (Received February 28,1950 .)

329t. L. A. Zadeh: The correlation function of the response of a variable system.

Consider a linear varying-parameter system in which one or more parameters are stationary functions of time. In a recent paper (Proceedings of the I.R.E. vol. 38 (1950) pp. 291-299) the system function of a linear varying-parameter system was defined as a function $H(j \omega ; t)$ such that $H(j \omega ; t) e^{j \omega t}$ is the response of the system to an input of the form $e^{j \omega t}$. Define the correlation function of the system as $\psi(\tau ; \omega)$ $=\overline{H(j \omega ; t) H(-j \omega ; t+\tau)}$. Let the input to the system be stationary and uncorrelated with $H(j \omega ; t)$, and let its spectral density be $S(\omega)$. It is shown that the correlation function of the response of the system to this input is given by the relation $\psi(\tau)$ 
$=(2 \pi)^{-1} \int_{-\infty}^{\infty} \psi(\tau ; \omega) S(\omega) e^{j \omega \tau} d \omega$. This result may be stated in the form of a theorem as follows: The correlation functions of the input and the output of a variable system $N$ may be regarded as the input and output of variable system $N^{*}$ whose system function is the correlation function of the system function of $N$. This theorem has numerous practical applications. (Received March 15, 1950.)

\section{0t. Othar Zaldastani: Operator methods in the theory of one-} dimensional unsteady compressible fluid flow.

If the ratio, $\kappa$, of specific heats equals $(2 N+1) /(2 N-1), N$ being an integer, the nonlinear partial differential equations of the unsteady rectilinear motion of a compressible fluid can be solved by using the Legendre transform $U$ of the stream function. Using this procedure, the author extends the results of Beckert (Annals of Physics vol. 5 (1940) p. 38). In particular, he gives general equations for the particle lines in the $u, v$-plane, $u$ being the velocity and $v$ a function of the velocity. For $\kappa=1.4$, the problem of the study of the flows is reduced to the integration of $U_{u u}-U_{v v}$ $=-4 U_{v} / v$, for which the general solution $U=\int v \int v(f+g) d v d v$ is used, where $f$ and $g$ are arbitrary functions respectively of $u+v$ and $u-v$. Bergman's integral operator of the second kind (Bergman, Proceedings of the First Symposium in Applied Mathematics, American Mathematical Society, 1949, pp. 19-40) supplies another form of a general solution, which is particularly useful in applications, since the generating function of the later operator (for $N$ integer) becomes a Bernoulli polynomial. Several initial and boundary value problems are considered, and a complete study of the continuous motion of a fluid between the fixed pistons is given for $\kappa=1.4$. (Received March 30, 1950.)

\section{GEOMETRY}

\section{H. S. M. Coxeter: Integral Lorentz transformations.}

An integral Lorentz transformation is a linear transformation with integral coeffcients leaving the form $t^{2}-x^{2}-y^{2}-z^{2}$ invariant. It is known (see A. Schild, Canadian Journal of Mathematics vol. 1 (1949) p. 47) that the group of all such transformations is generated by the changes of sign of the coordinates, the permutations of $x, y, z$, and the reflection that changes $(r, x, y, z)$ into $(2 t-x-y-z, t-y-z, t-z-x, t-x-y)$. The transforms of $(1,0,0,0)$ are the integral solutions of the Diophantine equation $t^{2}-x^{2}-y^{2}-z^{2}=1$. When regarded as points in hyperbolic space with the absolute quadric $t^{2}-x^{2}-y^{2}-z^{2}=0$, these are the vertices of the hyperbolic honeycomb whose Schläfli symbol is $\{4,4,3\}$. (In the same notation the ordinary cubic lattice in Euclidean space is $\{4,3,4\}$.) (Received March 6, 1950.)

\section{2t. Jack Levine: Collineations in generalized spaces. II.}

It is shown that the equations $\Delta \mathrm{I}_{j k}^{i}=0$ (Buchin Su, Descriptive collineations in spaces of $K$-spreads, Trans. Amer. Math. Soc. vol. 61 (1947) pp. 495-507) defining the projective collineations of a generalized space $H_{n}$ can be expressed in the equivalent simpler form $\Delta P^{i}=0$ (where $\Delta$ is the Lie derivative operator). The components $\Pi_{j k}^{i}$ of projective connection are obtained in the form $2 \Pi_{j k}^{i}=\partial^{2} P^{i} / \partial p^{i} \partial p^{k}$, and $P^{i}$ $\equiv H^{i}-p^{i} F /(n+1), \quad F=\partial H^{i} / \partial p^{i}$. The paths of an $H_{n}$ are given by $d^{2} x^{i} / d t^{2}$ $+\Pi_{j k}^{i}(x, p) p^{i} p^{k}+p^{i} F(x, p) /(n+1)=0$, where $p^{i}=d x^{i} / d t$. The $P^{i}$ satisfy the identity $\sum \partial P^{i} / \partial x^{i}=0$. All generalized spaces of two dimensions admitting real groups $G_{r}$ of projective collineations are determined by solving the equations $\Delta P^{i}=0$. There are nine such types of $H_{2}$ and $r \leqq 3$. (Received March 15, 1950.) 
333. T. S. Motzkin: Duality for general varieties. Preliminary report.

Let $p \neq 0$ be the characteristic of the (algebraically closed) coordinate field. For a hypersurface whose general point $P$ is not parabolic, duality is birational and involutory. For other hypersurfaces the tangent hyperplane $T$ at $P$ touches with multiplicity 2 or $p^{e}$; in both cases the dual may have, or not have, smaller dimension; and in the latter case the variety of points whose tangent hyperplane is $T$ need not be linear, nor split into linear varieties. For a surface in 6-space duality may be noninvolutory even if it is birational and even if $p=0$. For curves, cf. Bull. Amer. Math. Soc. Abstracts 56-1-25, 56-3-193, 56-3-194. Illustrations are given by monomial varieties $x_{i}=c_{i} \prod t_{k}^{a} 2 k$, other abelian varieties, and Fermat hypersurfaces $\sum x_{i}^{n}=0$. (Received May 1, 1950.)

\section{Statistics ANd Probability}

334t. K. L. Chung and Mark Kac: Remarks on fluctuations of sums of independent random variables.

Let $X_{1}, X_{2}, \cdots$ be independent random variables each having the same symmetric stable distribution with exponent $\alpha(0<\alpha \leqq 2)$. Let $s_{k}=X_{1}+\cdots+X_{k}$ and denote by $N_{n}$ the number of changes of $\operatorname{sign}$ in the finite sequence $s_{1}, s_{2}, \cdots, s_{n}$. For $1<\alpha \leqq 2$ the limiting distribution of $N_{n} / n^{1-1 / \alpha}$ is determined explicitly and the answer is related to the Mittag-Leffler function $E_{a}(-x)$. For $0<\alpha<1$ the limiting distribution of $N_{n} / \log n$ is proved to be exponential with the exponent depending on $\alpha$. For $\alpha=1$ (Cauchy distribution) the limiting distribution of $N_{n} /\left(2 \pi^{2}(\log n)^{2}\right.$ is exponential $\left(1-e^{-x}\right)$. (Received February 16, 1950.)

\section{Mark Kac and Murray Rosenblatt: On diffusion with $a b$ -} sorbing barriers in more than 1 dimension.

Let $\bar{r}(t)$ be an element of the product space of $n$ independent Wiener spaces. Let $C$ be the bounding surface of the region $R$ in the space of $n$-vectors $r$. It is assumed that $C$ satisfies the regularity conditions of Poincaré and that $R$ contains the origin. The probability $u(\bar{x}, t)=\operatorname{Pr}(\bar{r}(t)=\bar{x}, \bar{r}(\tau) \in R, 0 \leqq \tau<t)$ is shown to be the fundamental solution of $\partial u / \partial t=\nabla^{2} u / 2$ with boundary value zero at $C$ and singularity at the origin. Let $V(\bar{r})$ be the characteristic function of the complement of $R$. The expectation $E\left(\exp \left(-u \int_{0}^{t} V(\bar{r}(\tau)) d \tau\right) \mid \bar{r}(t)=\bar{x}\right) \exp \left(-\bar{x}^{2} / 2 t\right)(2 \pi t)^{-n / 2}$ is considered. The derivation uses the fact that $u(x, t)=\lim _{u \rightarrow \infty} \exp \left(-\bar{x}^{2} / 2 t\right)(2 \pi t)^{-n / 2} E\left(\exp \left(-u \int_{0}^{t} V(r(\tau)) d \tau\right) \mid \bar{r}(t)\right.$ $=\bar{x}$ ) and the method of barriers. (Received April 28, 1950.)

\section{6t. C. N. Mooers: Logic of selective systems.}

In the digital description and selection of non-numerical information, one technique (Zatocoding) employs a collection of information carriers, such as cards or frames of film strip, each bearing a multiposition digital symbol field with each position taking a mark or blank, assigns a random pattern of marks in the field to each descriptor of information, and combines the several patterns in each field by Boolean addition. Descriptor patterns defining a selection of cards are combined by Boolean addition in the selector, and selection occurs when the total pattern in the field includes the total selector pattern. Aside from the statistically-expected "noise" in the selection, selection is found to be according to the logical product of the selector de- 
scriptors. One-step selection according to logical sum is found to be incompatible with adequate control of "noise" for all variations by complementation or duality when pattern inclusion rules the selections. (Received March 15, 1950.)

\section{TOPOLOGY}

\section{7t. R. D. Anderson: On collections of pseudo-arcs.}

The author studies collections of pseudo-arcs. Bing has recently shown that if $H$ and $K$ are chained hereditarily indecomposable continua, $H$ is topologically equivalent to $K$. The pseudo-arc of Moise is, by construction, a chained hereditarily indecomposable continuum. The author shows, using Bing's result, that there exists a continuous collection of pseudo-arcs filling up the plane. It is known that such a collection must be topologically equivalent to the plane. In demonstrating the above theorem the author proves a lemma which implies, among other things, that there exists a continuous collection of pseudo-arcs filling up a closed annulus such that this collection is topologically equivalent to a simple closed curve. (Received March 14, 1950.)

\section{R. H. Bing and E. E. Floyd: Coverings with connected inter- sections.}

Suppose $S$ is a compact locally connected metric continuum. There is a countable basis for $S$ such that the common part of the elements of any subcollection of this basis is connected and uniformly locally connected. Furthermore, the collection of closures of the elements of this basis is a family $G$ of continuous curves such that the common part of the elements of any subcollection of $G$ is either null or a continuous curve. (This is an extension of a result of $\mathrm{R}$. D. Anderson [Concerning upper semicontinuous collections of continua, Trans. Amer. Math. Soc. vol. 67 (1949) pp. 451460 ].) These results are proved by making use of brick partitionings of $S$. (Received March 7, 1950.)

339. V. L. Klee: $A$ proof that Hilbert space is homeomorphic with the surface of its unit sphere.

Suppose that $E$ is a nonreflexive normed linear space. By use of the fact that $E$ must contain a decreasing sequence of bounded closed convex sets whose intersection is empty, it is proved that (a) every hyperplane in $E$ is homeomorphic with the surface of the unit sphere of $E$; (b) there is a homeomorphism of period 2 without fixed points of $E$ onto itself which takes the (solid) unit sphere onto itself. These statements also apply to many reflexive spaces, and in particular to Hilbert space (since $\left(l^{1}\right)$ is homeomorphic with $\left.\left(l^{2}\right)\right)$. Thus certain questions of S. Kakutani and P. A. Smith are answered. (Received March 14, 1950.)

340t. J. E. L. Peck: The embedding of topological groupoids in topoTogical quasigroups.

An alternation topological groupoid is a topological set, closed under a binary, continuous operation, in which any four elements satisfy the relation $(a b)(c d)$ $=(a c)(b d)$. An element is a $*$ canceller if all its powers may be cancelled on both sides. A topological quasigroup is a topological groupoid in which any one of the three elements, related by $a=b c$, is a unique, continuous function of the other two. It is proved 
that an alternation topological groupoid, in which the set of *cancellers is open and in which the operation satisfies a further continuity restriction, may be embedded in an alternation topological groupoid, whose set of *cancellers form a topological quasigroup, containing the original *cancellers. This topological theorem is based on the algebraic results of Scholander (Bull. Amer. Math. Soc. vol. 55 (1949) pp. 746-757). A similar theorem is proved for noncommutative topological semigroups, using the algebraic results of Dubreil (Algèbre, Paris, 1946). It is also shown that a compact topological semigroup, with a cancellation law, is a topological group. (Received April 17, 1950.)

H. M. MACNEILle, Executive Director 